\title{
Distracting your enemy with bubbles
}

4

exosomes

function as

decoys that

neutralize

bacterial

pore-forming

toxins to

protect host

cells
Many pathogenic bacteria secrete pore-forming toxins as potent virulence factors. These toxins perforate host cell membranes, causing lysis of their target cells. Given the major role of pore-forming toxins in pathogenicity, host cells need to protect themselves from the toxic effects. In a new study, Torres, Cadwell and colleagues provide insights into a mechanism whereby host cells deploy extracellular vesicles as decoys that trap and 'neutralize' Staphylococcus aureus $\alpha$-toxin (also known as a-haemolysin) to avoid cell damage.

Autophagy is an evolutionary conserved cellular process whereby double-membrane vesicles (termed autophagosomes) engulf and deliver intracellular material and invading pathogens to the lysosome for degradation. Autophagy-related protein ATG16L1 is a host protein that is involved in autophagosome formation. The authors have previously shown that ATG16L1depleted (and thus autophagydeficient) mice exhibit increased susceptibility to methicillin-resistant $S$. aureus strains that encode a-toxin, a finding that implicates

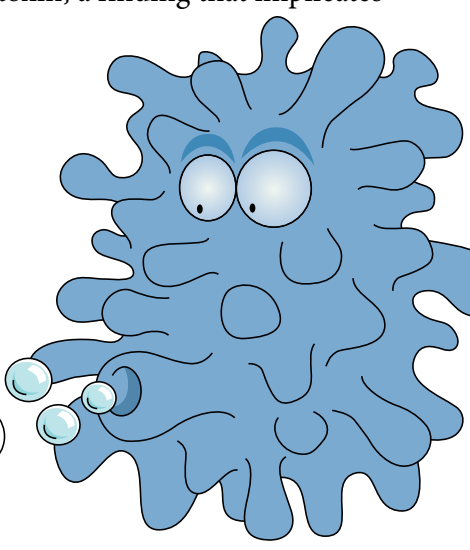

ATG16L1 in the protection against the pathogen.

Bacterial $\alpha$-toxin binds to host cell membranes through the metalloprotease ADAM10. Now, the authors report that depletion of ATG16L1 in a human alveolar epithelial cell line led to an increase in the levels of cell surface and total ADAM10, thus linking the presence of the autophagy protein to reduced receptor levels on the cell surface.

But how does the autophagy machinery decrease the levels of ADAM10 on the cell surface? In addition to a role in conventional autophagy, ATG proteins have nonconventional functions, such as controlling extracellular secretion. This, together with the previous finding that ADAM10 is incorporated into exosomes (which are endosome-derived extracellular vesicles), led the authors to hypothesize that ATG proteins induce the secretion of ADAM10 on exosomes to prevent its accumulation on the cell surface. They found that knockdown of ATG16L1 reduced the total number of ADAM10-positive exosomes in the culture supernatant as well as in the blood from ATG16L1-deficient mutant mice, whereas the amount of ADAM10 per exosome was unaffected. This finding suggests that the ATG proteins regulate exosome biogenesis and not the incorporation of the substrate into exosomes.

Next, the authors went on to show that the production of exosomes was induced by the presence of bacteria. Specifically, they found that bacterial DNA and CpG DNA induce the biogenesis Credit: Philip Patenall/Springer Nature Limited of exosomes.
Furthermore, the authors isolated exosomes from the supernatant of donor cells and reported that those exosomes were able to protect recipient epithelial cells that have been treated with $\alpha$-toxin. By contrast, exosomes isolated from ATG16L1-knockdown cells did not exhibit a protective effect. Similarly, exosomes isolated from ADAM10-knockdown cells did not protect cells from cell death induced by a-toxin. Further experiments showed that the toxin assembles into oligomers on exosome membranes, which is the configuration that forms a pore in the target cell membrane.

To test the protective effect in vivo, the authors transferred exosomes from wild-type or Atg16l1-deficient mice into recipient mice and infected the animals with a lethal dose of S. aureus. Transfer of the exosomes from wild-type mice, but not from mutant mice, increased the survival of the infected recipient mice. In addition, exosomes from wildtype mice improved survival of Atg16l1-deficient mice, which suggests that the observed increased susceptibility of autophagy-deficient mutants is in part due to the reduced production of exosomes.

In summary, the findings of this study suggest that exosomes function as decoys that neutralize bacterial pore-forming toxins to protect host cells from membrane damage and lysis. The authors suggest that such 'defensosomes' may form a previously unknown innate immune response to bacterial infection.

Andrea Du Toit

ORIGINAL ARTICLE Keller, M. D. et al. Decoy exosomes provide protection against bacterial toxins. Nature 579, 260-264 (2020) 